\title{
State of the debate contingent arguments
}

\author{
Article
}

Accepted Version

Creative Commons: Attribution-Noncommercial-No Derivative Works 4.0

Felgenhauer, M. and Xu, F. (2019) State of the debate contingent arguments. Economics Letters, 179. pp. 46-48. ISSN 0165-1765 doi:

https://doi.org/10.1016/j.econlet.2019.03.027 Available at https://centaur.reading.ac.uk/86004/

It is advisable to refer to the publisher's version if you intend to cite from the work. See Guidance on citing.

To link to this article DOI: http://dx.doi.org/10.1016/j.econlet.2019.03.027

Publisher: Elsevier

All outputs in CentAUR are protected by Intellectual Property Rights law, including copyright law. Copyright and IPR is retained by the creators or other copyright holders. Terms and conditions for use of this material are defined in the End User Agreement.

\section{www.reading.ac.uk/centaur}

\section{CentAUR}

Central Archive at the University of Reading

Reading's research outputs online 


\title{
State of the debate contingent arguments
}

\section{MIKE FELGENHAUER ${ }^{1}$}

Plymouth University

\author{
FANGYA XU²
}

Plymouth University

March 19, 2019

\begin{abstract}
This paper studies the informative content of controversial scientific arguments depending on the state of the debate. Researchers are assumed to differ in their degree of opportunism. The arguments considered are manipulable, may stem from sequential private experimentation and can be revealed selectively. Arguments of opportunistic researchers tend to be informative if there is a lack of consensus in the debate and uninformative if it is more settled. Arguments of more sincere researchers may be uninformative if there is a lack of consensus in the debate and informative if it is more settled. The effect of institutional incentives on the informativeness of arguments should depend on the state of the debate.
\end{abstract}

Keywords: Persuasion, experimentation, manipulation.

JEL classification: D82, D83

https://doi.org/10.1016/j.econlet.2019.03.027

@ 2019. This manuscript is made available under the CC-BY-NC-ND 4.0 license

http://creativecommons.org./licenses/by-nc-nd/4.0/

${ }^{1}$ Correspondence address: Plymouth University, Cookworthy Building, Plymouth, PL48AA, UK; mike.felgenhauer@plymouth.ac.uk.

2 fangya.xu@plymouth.ac.uk 


\section{Introduction}

This paper studies the informative content of arguments in scientific debates depending on the state of the debate. We assume that researchers differ in their degree of opportunism, i.e., how much they care about a publication even if it does not reflect the truth. In scientific debates arguments tend to be published sequentially. We assume that a paper is published if it is surprising in the sense that it goes against the state of the debate. That is, the arguments are controversial. We compare the informative content of arguments if there is a lack of consensus in the debate and where it is more settled. There is a lack of consensus if it is not considered as obvious what the "truth" is. There is a consensus if researchers tend to agree on what the truth is.

We consider arguments that are manipulable, that may stem from sequential private experimentation and that are revealed selectively. Consider, e.g., regressions on a privately collected database. If the database is not manipulated, then each regression can be viewed as an experiment that yields an informative outcome in favour or against a hypothesis. The decision to continue to run regressions is sequential, as it depends on the experimentation history. Regressions are run privately and the outcomes can be reported selectively. In addition to running informative regressions on a non-manipulated database, uninformative manipulation of the data is possible such that a particular regression on these data yields the desired outcome.

We find that the benefit from manipulation for opportunistic researchers decreases slower in the degree of consensus in the debate than the benefit from experimenting without manipulation. If there is a lack of consensus, then these types prefer experimentation, as an experiment is relatively likely to yield a surprising outcome. In this case, their arguments are informative. If there is a consensus instead, then they prefer manipulation, as it becomes unlikely to find a surprising outcome given the adverse state of the debate. For more sincere researchers the effects are reversed. Such a type does not want to manipulate if there is a consensus, as he does not like a publication that is likely to be false. If there is a lack of consensus, and he is less certain about the truth, then manipulation may dominate experimentation. Institutional incentives, such as publication based rewards, should affect how opportunistic researchers are. The effect of such incentives on the informativeness of scientific arguments, thus, also depends on the state of the debate. Finally, we find that the editor/receiver-preferred design of research methods should depend on the degree of consensus. For example, a method that could reveal the truth with certainty is in general not receiverpreferred for all states of the debate.

This paper is part of the literature on persuasion with information acquisition. Kamenica and Gentzkow (2011) and Felgenhauer and Loerke (2017) consider the design of a non-manipulable experiment under public and sequential private experimentation, respectively. The design depends on the prior belief in both settings. Felgenhauer and Schulte (2014) consider a fixed design of non-manipulable experiments and focus on how many experiments are run in private. The incentive to experiment (excessively) is reduced the more extreme the prior belief is, i.e., the more consensus there is in our sense, since it becomes less likely to find a sufficient number of positive outcomes for persuasion soon enough to justify experimentation costs. Felgenhauer and $\mathrm{Xu}$ (2018) study the effect of a change of the face value of an argument on its real value if the argument stems from sequential private experimentation with and without manipulation. The current model builds on Felgenhauer and $\mathrm{Xu}$ (2018), but we focus on whether manipulation or experimentation is optimal depending on the state of the debate. 


\section{Model}

There is an unknown state of the world $\omega \in\left\{\omega_{1}, \omega_{2}\right\}$ with the prior belief $\operatorname{prob}\left\{\omega=\omega_{1}\right\}=\mu, \mu \in$ $\left(0, \frac{1}{2}\right)$, where $\mu$ is interpreted as the state of the debate. The degree of consensus decreases in $\mu$.

There is a sender (researcher) and a receiver (editor). The receiver chooses action $a \in\left\{a_{1}, a_{2}\right\}$. The sender's gross utility is as follows:

\begin{tabular}{|c|c|c|}
\hline & $\omega=\omega_{1}$ & $\omega=\omega_{2}$ \\
\hline $\mathrm{a}=\mathrm{a}_{1}$ & 1 & $\theta$ \\
\hline $\mathrm{a}=\mathrm{a}_{2}$ & 0 & 0 \\
\hline
\end{tabular}

The sender's type is $\theta \in[-1,1]$. In an academic context $\theta$ indicates the opportunism of a researcher. ${ }^{3}$ If $\theta=1$, then the researcher exclusively cares about a publication $\left(a=a_{1}\right)$ regardless of whether the results truly reflect the state of the world. As $\theta$ decreases, he feels less comfortable with a publication if the results published are "false" than if they are "true".

The sender may sequentially run experiments or manipulate. Each experiment costs $c_{\mathrm{E}}>0$ and yields an outcome $s \in\left\{s_{1}, s_{2}\right\} . s_{1}$ denotes a positive outcome and $s_{2}$ an adverse outcome. Suppose $\operatorname{prob}\left\{s=s_{1} \mid \omega=\omega_{1}\right\}=\operatorname{prob}\left\{s=s_{2} \mid \omega=\omega_{2}\right\}=1$ for simplicity. This assumption implies that there is at most one experiment at the optimum. Manipulation costs $c_{M}>0$ and yields $s_{1}$ with certainty. Costs are subtracted from the gross utility.

The sender sends message $m \in\left\{s_{1}, s_{2}, \varnothing\right\}$. Message $m \in\left\{s_{1}, s_{2}\right\}$ is only possible if the corresponding outcome is obtained via experimentation or manipulation. The receiver mechanically chooses $\mathrm{a}=\mathrm{a}_{1}$ if she observes $\mathrm{m}=\mathrm{s}_{1}$ and $\mathrm{a}=\mathrm{a}_{2}$ otherwise. The receiver's behaviour is endogenized in the Discussion. As $\mu \in\left(0, \frac{1}{2}\right)$, in the academic context, the editor only publishes a result that challenges the state of the debate.

The timing is as follows. The sender sequentially chooses whether to experiment, manipulate or to do nothing. He then sends $m \in\left\{s_{1}, s_{2}, \varnothing\right\}$ to the receiver. Finally, the receiver chooses a $\in$ $\left\{\mathrm{a}_{1}, \mathrm{a}_{2}\right\}$ and the game concludes.

\section{Analysis}

The benefit from running the first experiment and then stopping without manipulation is $\mu-$ $c_{E}$, with $\mu-c_{E}>0 \Leftrightarrow \mu>c_{E}$ and $\frac{d\left(\mu-c_{E}\right)}{d \mu}=1$. The benefit from manipulating at the outset is $\mu+$ $(1-\mu) \theta-c_{M}$, with $\mu+(1-\mu) \theta-c_{M}>0 \Leftrightarrow \mu>\frac{c_{M}-\theta}{1-\theta}$ and $\frac{d\left(\mu+(1-\mu) \theta-c_{M}\right)}{d \mu}=1-\theta$. Both of these benefits are lines with respect to $\mu$. The benefits are equal if $\mu-c_{E}=\mu+(1-\mu) \theta-c_{M} \Leftrightarrow \mu=$ $1-\frac{\mathrm{c}_{\mathrm{M}}-\mathrm{c}_{\mathrm{E}}}{\theta}$. Suppose $1-\frac{\mathrm{c}_{\mathrm{M}}-\mathrm{c}_{\mathrm{E}}}{\theta} \in\left(0, \frac{1}{2}\right)$.

The benefit from manipulating after observing an adverse outcome of the first experiment (in which case the state $\omega=\omega_{2}$ is obvious) is $\theta-c_{M}$. If $\theta-c_{M}>0$, then manipulation is optimal either at the outset or after observing an adverse experimental outcome. For these parameters a positive outcome is uninformative. Suppose in the following $\theta-c_{M}<0$, i.e., the type does not manipulate after observing an adverse outcome of an experiment.

Proposition 1 (i) Let $\theta>0$ and $\frac{\mathrm{c}_{\mathrm{M}}-\theta}{1-\theta}<1-\frac{\mathrm{c}_{\mathrm{M}}-\mathrm{c}_{\mathrm{E}}}{\theta}$. Message $\mathrm{m}=\mathrm{s}_{1}$ is uninformative if $\mu \in\left[\frac{\mathrm{c}_{\mathrm{M}}-\theta}{1-\theta}, 1-\right.$ $\left.\frac{c_{M}-c_{E}}{\theta}\right)$. Message $m \in\left\{s_{1}, s_{2}, \varnothing\right\}$ reveals state $\omega$ if $\mu \in\left[1-\frac{c_{M}-c_{E}}{\theta}, \frac{1}{2}\right)$. There is no experimentation

\footnotetext{
${ }^{3}$ The replication crisis and institutional features, like single/double blind refereeing, suggest that opportunism matters in academia.
} 
or manipulation if $\mu<\frac{c_{M}-\theta}{1-\theta}$. (ii) Let $\theta<0$ and $c_{E}<1-\frac{c_{M}-c_{E}}{\theta}$. Message $m \in\left\{s_{1}, s_{2}, \varnothing\right\}$ reveals state $\omega$ if $\mu \in\left[c_{E}, 1-\frac{c_{M}-c_{E}}{\theta}\right]$. Message $m=s_{1}$ is uninformative if $\mu \in\left(1-\frac{c_{M}-c_{E}}{\theta}, \frac{1}{2}\right)$. There is no experimentation or manipulation if $\mu<\mathrm{c}_{\mathrm{E}}$.

Proof: (i) $\theta>0$ ensures that the benefit from running an experiment without manipulation increases faster in $\mu$ than the benefit from manipulation at the outset. $\mu \geq \frac{c_{M}-\theta}{1-\theta}$ ensures that the benefit from manipulation is non-negative. If $\mu<1-\frac{c_{M}-c_{E}}{\theta}$, then the benefit from manipulation at the outset exceeds the benefit from experimentation, rendering a positive outcome uninformative. If $\mu \in\left[1-\frac{\mathrm{c}_{\mathrm{M}}-\mathrm{c}_{\mathrm{E}}}{\theta}, \frac{1}{2}\right)$, then the sender prefers experimentation without manipulation and any outcome (or not providing an outcome) is informative.

(ii) $\theta<0$ ensures that the benefit from running an experiment without manipulation increases slower in $\mu$ than the benefit from manipulation at the outset. $\mu \geq c_{\mathrm{E}}$ ensures that the benefit from experimentation without manipulation is non-negative. If $\mu \in\left[c_{E}, 1-\frac{c_{M}-c_{E}}{\theta}\right]$, then the benefit from experimentation without manipulation exceeds the benefit from manipulation at the outset, rendering any outcome (or not providing an outcome) informative. For larger $\mu$, the sender prefers manipulation and a positive outcome is uninformative.

Parameters exist for which all conditions are satisfied. Q.E.D.

The probability with which experimentation yields a positive outcome $s=s_{1}$ depends on the state of the debate $\mu$. This probability increases in $\mu$. Manipulation yields $s_{1}$ with certainty.

A sincere researcher $(\theta<0)$ does not like to manipulate if there is more consensus $($ low $\mu)$, as it likely yields $s_{1}$ in the wrong state $\omega=\omega_{2}$. Experimentation does not have this drawback. If there is a lack of consensus (high $\mu$ ), then it is less likely that manipulation yields $s_{1}$ in the wrong state $\omega=\omega_{2}$. Manipulation then dominates experimentation if manipulation costs are relatively low.

An opportunistic researcher $(\theta>0)$ prefers manipulation if there is more consensus (low $\mu)$. He does not care that much about the state $\omega$. Experimentation yields a positive outcome with a low probability and manipulation yields such an outcome with certainty. If there is a lack of consensus (high $\mu$ ), then there is a higher chance to obtain a positive outcome by experimentation. If experimentation costs are sufficiently low, then experimentation dominates manipulation in this case.

\section{Discussion}

Instead of an exogenous decision rule, suppose that the receiver has a threshold of doubt $\mathrm{p}_{\mathrm{d}} \in$ $(\mu, 1)$, which implies that at the optimum she only chooses the sender's preferred action if $\operatorname{prob}\left\{\omega=\omega_{1} \mid m=s_{1}\right\} \geq p_{d}$. In such a model, the receiver does not choose in favour of the sender if she deduces that manipulation occured. This renders costly manipulation worse than not doing anything. The sender also cannot commit to experiment. Hence, for parameters where manipulation is optimal above, it can be easily shown that there is no equilibrium with persuasion if the sender's type is known. If there is a non-trivial distribution of privately known sender types, then it is straightforward to show that there can be equilibria with persuasion, where some types experiment and others manipulate. Manipulation has a negative impact on $\operatorname{prob}\left\{\omega=\omega_{1} \mid \mathrm{m}=\mathrm{s}_{1}\right\}$. The impact of experimentation on $\operatorname{prob}\left\{\omega=\omega_{1} \mid \mathrm{m}=\mathrm{s}_{1}\right\}$ is positive. An equilibrium with persuasion exists if the threshold of doubt is sufficiently low. The state of the debate affects which types manipulate or experiment. 
$\theta$ reflects a researcher's opportunism. $\theta$ could be affected by publication pressure due to institutional incentives. As the researcher's behaviour depends on $\theta$, the impact of institutional incentives depends on the state of the debate.

We assume that an experiment perfectly predicts the state of the world. Consider parameters, where manipulation is optimal for a type in the model in section 3, e.g., a high degree of consensus and an opportunistic researcher. A positive outcome is then uninformative. An experiment that does not perfectly predict the state of the world, may induce experimentation for this researcher, as it may have a higher chance to yield a positive outcome given the adverse state of the debate. Hence, running this experiment may be more attractive than manipulation. For example, consider a lower quality design with $\operatorname{prob}\left\{s=s_{1} \mid \omega=\omega_{1}\right\}=1$ and $\operatorname{prob}\left\{s_{n}=s_{2} \mid \omega=\right.$ $\left.\omega_{2}\right\}<1$. With an exogenous decision rule, running such an experiment and stopping without manipulation is more attractive than with $\operatorname{prob}\left\{s=s_{2} \mid \omega=\omega_{2}\right\}=1$. Hence, for a state of the debate where uninformative manipulation is just better than experimentation with $\operatorname{prob}\left\{\mathrm{s}=\mathrm{s}_{2} \mid \omega=\omega_{2}\right\}=1$, such a lower quality design renders informative experimentation optimal. A receiver who is interested in an informative message, thus, may prefer a lower quality to a higher quality design depending on the state of the debate and the researcher type she is facing.

The current paper highlights the importance of the state of the debate on the value of arguments in a parsimonious model. In future work it would be interesting to determine the editor/receiver-preferred design of arguments depending on the degree of consensus. Another avenue is to study what kind of arguments are/should be chosen contingent on the state of the debate. For example, there are logical arguments that are acquired via sequential private draws of assumptions ("thought experiments"), where manipulation of logic and given assumptions is not possible. Finally, we suggest to link the micro analysis to the evolution of paradigms and scientific revolutions in the spirit of Kuhn (1970).

\section{References}

Felgenhauer, Mike, and Petra Loerke. 2017. "Bayesian Persuasion with Private Experimentation." International Economic Review, 58(3): 829-856.

Felgenhauer, Mike, and Elisabeth Schulte. 2014. "Strategic Private Experimentation." American Economic Journal: Microeconomics, 6: 74-105.

Felgenhauer, Mike, and Fangya Xu. 2018. "The (Face) Value of Arguments with and without Manipulation." Working paper.

Kamenica, Emir, and Matthew Gentzkow. 2011. "Bayesian Persuasion." American Economic Review, 101(6): 2590-2615.

Kuhn, Thomas S. 1970. "The Structure of Scientific Revolutions." Chicago: University of Chicago Press. 